\title{
The randomised uterine septum transsection trial (TRUST): design and protocol
}

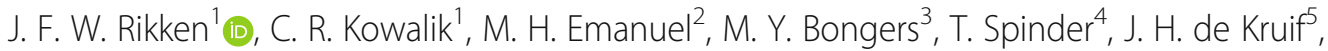 \\ K. W. M. Bloemenkamp ${ }^{2}$, F. W. Jansen', S. Veersema², A. G. M. G. J. Mulders' ${ }^{7}$, A. L. Thurkow ${ }^{1}$, K. Hald ${ }^{8}$, \\ A. Mohazzab ${ }^{9}$, Y. Khalaf ${ }^{10}$, T. J. Clark ${ }^{11}$, M. Farrugia ${ }^{12}$, H. A. van Vliet ${ }^{13}$, M. S. Stephenson ${ }^{14}$, F. van der Veen ${ }^{1}$, \\ M. van Wely ${ }^{1}$, B. W. J. Mol ${ }^{15}$ and M. Goddijn ${ }^{1 *}$
}

\begin{abstract}
Background: A septate uterus is a uterine anomaly that may affect reproductive outcome, and is associated with an increased risk for miscarriage, subfertility and preterm birth. Resection of the septum is subject of debate. There is no convincing evidence concerning its effectiveness and safety. This study aims to assess whether hysteroscopic septum resection improves reproductive outcome in women with a septate uterus.

Methods/design: A multi-centre randomised controlled trial comparing hysteroscopic septum resection and expectant management in women with recurrent miscarriage or subfertility and diagnosed with a septate uterus. The primary outcome is live birth, defined as the birth of a living foetus beyond 24 weeks of gestational age. Secondary outcomes are ongoing pregnancy, clinical pregnancy, miscarriage and complications following hysteroscopic septum resection. The analysis will be performed according to the intention to treat principle. KaplanMeier curves will be constructed, estimating the cumulative probability of conception leading to live birth rate over time. Based on retrospective studies, we anticipate an improvement of the live birth rate from $35 \%$ without surgery to $70 \%$ with surgery. To demonstrate this difference, 68 women need to be randomised.
\end{abstract}

Discussion: Hysteroscopic septum resection is worldwide considered as a standard procedure in women with a septate uterus. Solid evidence for this recommendation is lacking and data from randomised trials is urgently needed.

Trial registration: Dutch trial registry (NTR1676, 18th of February 2009).

Keywords: Septate uterus, Recurrent miscarriage, Subfertility, Hysteroscopic septum resection, Randomised controlled trial

\section{Background}

A septate uterus is a congenital uterine anomaly where the uterus is divided into two cavities. A septate uterus is associated with reduced fertility (RR 0.86 ; $95 \%$ CI $0.77-$ 0.96), increased miscarriage rates (RR 2.9; 95\% CI 2.0-4.1) and preterm delivery (RR 2.1; 95\% CI 1.5-3.1) [1].

The mechanisms behind the negative effect of the septum on fertility and pregnancy outcome have not yet been clarified. Suggestions are that the septum is a poor

\footnotetext{
* Correspondence: m.goddijn@amc.uva.nl

${ }^{1}$ Center for Reproductive Medicine, Academic Medical Centre, University of Amsterdam, PO Box 22700, 1100, DE, Amsterdam, The Netherlands Full list of author information is available at the end of the article
}

site for embryonic implantation due to the assumed poor vascularization, decreased sensitivity to preovulatory changes of the endometrium overlying the septum, uncoordinated contractility of the septum, or a local defect of vascular endothelial growth factor (VEGF) receptors in the endometrium covering the septal area [2-5]. A septate uterus is usually ascertained through recurrent pregnancy loss or subfertility, and occasionally by other complaints such as dysmenorrhoea or preterm birth. Approximately $6 \%$ of women with recurrent miscarriage and 3.5-6.4\% of subfertile women, has a septate uterus [6-8]. .In comparison, this prevalence is $2-3 \%$ in the general female population $[6,7]$. 
Although evidence for the effectiveness of resecting the uterine septum is limited, resection is considered standard care in many countries. The evidence is mainly based upon retrospective studies, in which improved pregnancy chances and live birth rates after hysteroscopic septum resection have been suggested [9-17].

The major flaw in most of the abovementioned studies is obviously the before/after design, since this will always favour the tested intervention as the prognosis without the intervention is usually good $[18,19]$. At this moment, in view of the limited quality of the performed studies it thus remains unclear whether removal of the septum will eliminate negative effects -if any- of a septate uterus, and whether the procedure does not cause harmful side effects [20] .

To assess whether surgical intervention in women with (recurrent) miscarriage, subfertility or preterm birth and a septate uterus will improve reproductive outcome, we propose The Randomised Uterine Septum Trial (TRUST).

\section{Methods}

\section{Design}

The Randomised Uterine Septum Trial (TRUST) is a multicentre randomised controlled trial in women with a septate uterus (NTR 1676). Women are randomised to hysteroscopic septum resection or expectant management.
Recruitment has started since October 2009 and is ongoing.

\section{Trial population}

The study population consists of women of reproductive age with a septate uterus and a history of (recurrent) miscarriage, subfertility or preterm birth (Fig. 1). Women with a contraindication to surgery are excluded for the trial. When the study started in 2008, the study population consisted of women with recurrent miscarriage and a septate uterus. During the course of the trial we found out that it was difficult to recruit and randomize women for the study. Since essentially the population is women with a septate uterus and a wish to conceive, we decided to broaden the inclusion criteria to make an effort to identify these women more easily. Thus in 2011 we extended the inclusion criteria into women with a septate uterus and recurrent miscarriage and/or subfertility, and in 2015 the inclusion criteria were extended to women with (recurrent) miscarriage, subfertility or preterm birth. All amendments were approved by the ethical committee of the Academic Medical Centre (AMC), Amsterdam. Recurrent miscarriage is defined as two or more, not necessarily consecutive, pregnancy losses before 20 weeks of gestational age. Subfertility is defined as the inability to conceive for a

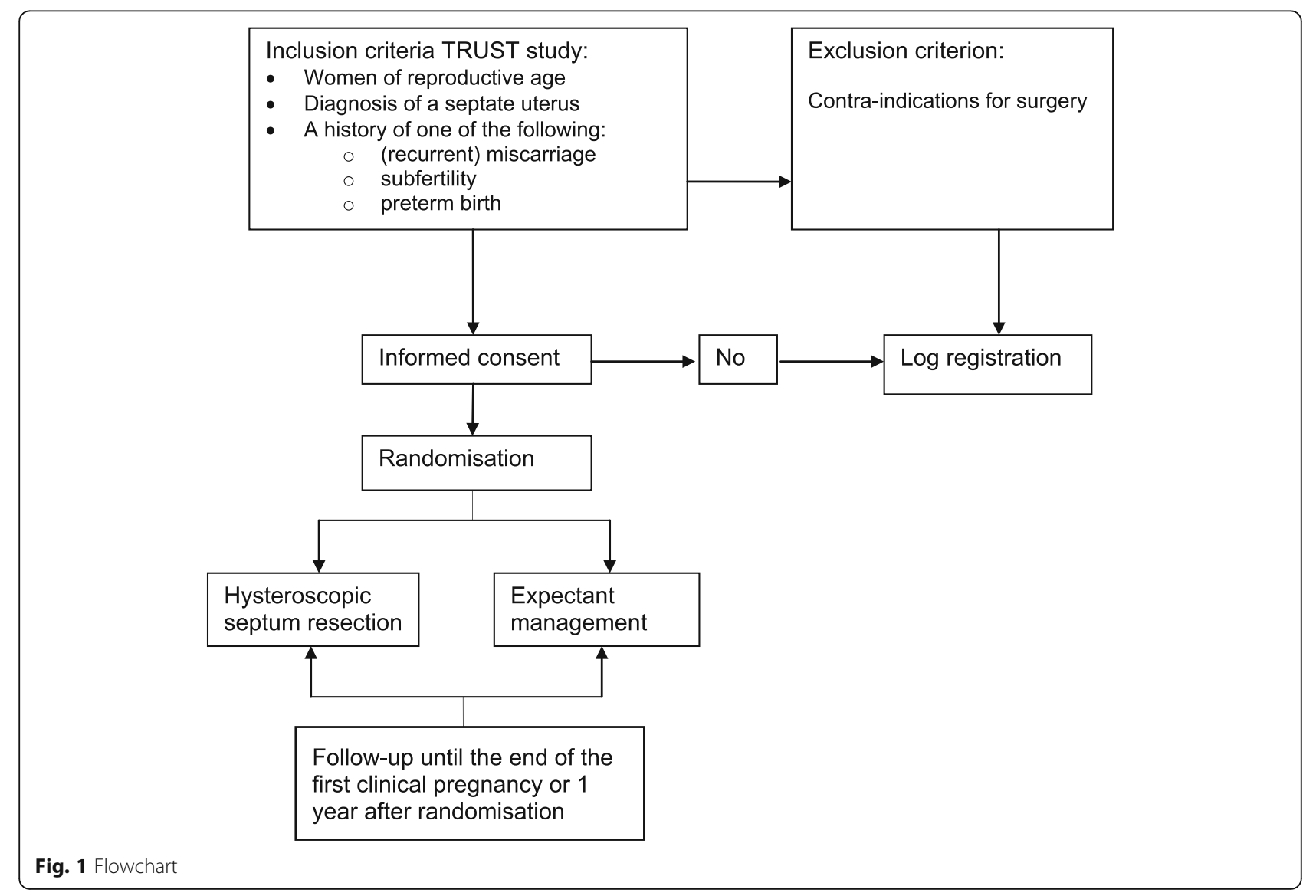


minimal period of one year of trying to conceive. The definition of preterm birth is birth before a gestational age of 37 complete weeks. Only women with an active wish to conceive are eligible for the trial. Following the most recent ESHRE-ESGE classification, a septate uterus is defined as all cases with an abnormal resorption of the midline septum, a normal outline of the uterus and an internal indentation at the fundal midline exceeding $50 \%$ of the uterine wall thickness regardless of the size of the septum [21]. Over the years there has been a lot of discussion about the classification system of uterine anomalies and ideas about the best definition of the septate uterus have changed [22]. After the following publication of the ESHRE-ESGE classification, we have adjusted the definition in the study protocol accordingly to these new insights, but it did not change our patient population [21]. The presence of a uterine septum is ascertained by HSG, 3D ultrasound (3D-US), MRI, saline or gel infusion sonohysterography or hysteroscopy combined with laparoscopy [23-26].

\section{Recruitment procedure}

Women are recruited from outpatient clinics located in the Netherlands, Iran and the United States of America. Women are invited to take part in the study when a septate uterus is diagnosed and when they meet the inclusion criteria.

\section{Ethics and trial registration}

Approval for this study and all subsequent amendments have been obtained from the Medical Ethical Committee of the Academic Medical Centre (IDS NL24082.018.08 MEC Academic Medical Centre, Amsterdam) The Netherlands. Local approval is obtained at all participating centres or will be obtained before the start of recruitment. The trial is registered within the Dutch trial registry (NTR1676).

Written informed consent is obtained from women fulfilling the inclusion criteria, prior to randomization.

\section{Randomization}

Once eligibility for the trial has been confirmed and women have given informed consent they are randomised via a web-based central randomization system to receive either hysteroscopic septum resection or expectant management .

\section{Hysteroscopic septum resection}

The intervention is hysteroscopic septum resection. The choice for an instrument and distension medium may vary per hospital and depends on the preference of the surgeon and instruments available. Analgesia during surgery can be either general anaesthesia or a loco-regional technique depending on the preference of the woman and whether concomitant surgery, in the form of laparoscopy, is scheduled.
To prevent uterine perforation during surgery, a laparoscopy or ultrasound has to be performed simultaneously [27]. The choice for laparoscopy or ultrasound depends on the local hospital protocol. To assess the results of the resection, a diagnostic hysteroscopy is performed 6-8 weeks postoperatively in an outpatient setting. When a secondary surgery is needed, this will be specified.

\section{Expectant management}

The control group will receive expectant management. Additional interventions, such as for example aspirin or heparin in case of co-existing antiphospholipid syndrome, or additional artificial reproductive techniques in women with subfertility, are allowed in both groups and will be registered. Should the first pregnancy after randomization result in a miscarriage, or should pregnancy not occur after one year of follow-up, women are free to opt for additional therapy, including hysteroscopic septum resection.

\section{Outcome measures}

The primary outcome measure is live birth, defined as the birth of a living foetus beyond 24 weeks of gestational age.

Secondary outcomes are ongoing pregnancy, clinical pregnancy, miscarriage, pregnancy outcomes as placental abruption, uterine rupture, preterm birth and mode of delivery (vaginal versus caesarean section), perinatal morbidity, mortality and complications following hysteroscopic septum resection, such as uterine perforation, fluid overload and endometritis.

\section{Follow up}

Follow up will take place for at least one year. Women who will conceive in that period will be followed for the course of that pregnancy. Woman allocated for expectant management, have the opportunity to opt for a hysteroscopic septum resection after one year of follow up, or when the next pregnancy results in a miscarriage. An additional timeline shows more detail on the timeline of study (see Additional file 1).

\section{Statistical analysis}

All analyses will be performed according to an intention to treat basis. The primary outcome, live birth, will be compared between the intervention and control group.

Relative risks and 95\% confidence intervals will be calculated for the relevant outcome measures. Time to conception, resulting in live birth will also be assessed by means of life table analysis. To estimate the cumulative probability of conception resulting in live birth rate over time Kaplan-Meier curves will be constructed. The risk of premature birth will be estimated stratified for gestational age. The relative risk for obstetrical and surgical complications will be calculated. 


\section{Sample size}

The sample size is based on retrospective studies, anticipating an improvement of the live birth rate from $35 \%$ without surgery to $70 \%$ with surgery. Using a two-sided test, an alpha-error of $5 \%$ and a beta-error of $20 \%$, two groups of 31 women are needed to demonstrate this difference. Anticipating lost-to-follow up and protocol violation, an additional $10 \%$ is needed. Thus, 68 women need to be randomised.

\section{Discussion}

Until this date, hysteroscopic septum resection of a septate uterus is standardly being performed worldwide in women of reproductive age and a wish to conceive [28, 29].

The evidence for this recommendation is based on retrospective and prospective comparative studies that suggest that restoration of the uterine morphology can potentially have a positive effect on live birth rate [9-17]. Most retrospective studies used their own participants as a control group. To our knowledge, over the years nine prospective comparative studies have been published. The studies describe miscarriage, pregnancy or live birth rate in women with a septate uterus who consented to hysteroscopic septum resection, compared with women who chose expectant management. These studies published contradictory findings, with some studies showing significant higher pregnancy rate in women with a septate uterus who were treated with surgery $[12,13,30,31]$, while other studies found no significant difference [32-35]. Thus, equipoise still exists and data from randomised trials are needed to provide definitive proof of effectiveness of a septum resection, or the lack thereof. In view of the major improvements in pregnancy rates reported by the non-comparative retrospective studies, we require a low number of participants to exclude a difference of $35 \%$ or more. By broadening our inclusion criteria we did not need to re-calculate the sample size since our primary population, i.e. women of reproductive age with a septate uterus, remained the same. Over the years, many studies have been published about the classification of uterine anomalies and the interrater disagreement in diagnosing the septate uterus still exists $[36,37]$. We have changed the definition of the septate uterus according to new insights leading to the ESHRE ESGE classification, and in retrospect all women meet these new criteria [21].

To our knowledge the TRUST trial is the first registered multi centre randomised controlled trial designed to assess whether hysteroscopic septum resection improves live birth rate (NTR1676). If hysteroscopic septum resection proves to be without effect on reproductive outcome, this would imply a major change in treatment policy and adapted guidelines.

\section{Additional file}

Additional file 1: Timeline of study. (PDF $12 \mathrm{~kb}$ )

\section{Abbreviations}

3D US: 3D Ultrasonography; TRUST: The Randomised Uterine

Septumtranssection Trial; VEGF: Vascular endothelial growth factor

\section{Acknowledgements}

The authors wish to thank the doctors and research nurses in the cooperating hospitals for all their effort.

Availability of data and materials

The datasets during and/or analyzed during the current study available from the corresponding author on reasonable request.

\section{Authors' contributions}

CRK drafted the first version of the paper, helped developing the protocol and was responsible for the logistical aspects of the start of the trial. JFWR further edited the paper and is currently responsible for the logistical aspects of the trial. MHE, MYB, TS, JHK, KWMB, FWJ, SV, AGMGJM, ALT and HAV managed the trial in the different hospitals in the Netherlands, and commented on the paper. AM is responsible for the trial in Iran and MSS in the USA, and both commented on the draft paper. KH, YK, MF, TJC, FV, MvW and BWJM contributed to the development of the protocol and commented on the draft paper. MG was responsible for the development of the protocol, had overall responsibility for the trial and commented on the draft paper. All authors read and approved the final paper.

\section{Ethics approval and consent to participate}

The TRUST study was approved by the Medical Ethical Committee of the Academic Medical Center Amsterdam at 28th of October 2008 (MEC 08/245). Written informed consent to participate in the study will be obtained from all participants.

\section{Consent for publication}

Not applicable.

\section{Competing interests}

The authors declare that they have no competing interests, with the exception of:

A.L. Thurkow:

Member International Surgical Advisory Board for Gynaecology Olympus.

Member International Advisory Board for Energy Devices(Division of Ethicon inc., Johnson \& Johnson).

Member Hologic International Advisory Board for minimal invasive procedures.

M.H. Emanuel:

Consultant and member medical advisory board Smith and Nephew Ltd. Inventor and royalty receiver hysteroscopic morcellation Smith and Nephew Ltd.

Inventor and royalty receiver gel instillation sonohysterography

GynaecologIQ BV.

S. Veersema:

Consultant at Bayer healthcare.

Member Hologic Advisory Board for minimal invasive procedures.

M.Y. Bongers:

Member Hologic Advisory Board for minimal invasive procedure

\section{Publisher's Note}

Springer Nature remains neutral with regard to jurisdictional claims in published maps and institutional affiliations.

\section{Author details}

${ }^{1}$ Center for Reproductive Medicine, Academic Medical Centre, University of Amsterdam, PO Box 22700, 1100, DE, Amsterdam, The Netherlands.

${ }^{2}$ University Medical Center Utrecht, Heidelberglaan 100, 3584 Utrecht, The Netherlands. ${ }^{3}$ Maxima Medical Centre, de Run 4600, 5504, DB, Veldhoven, 
The Netherlands. ${ }^{4}$ Leeuwarden Medical Centre, Henri Dunantweg 2, 8934, AD, Leeuwarden, the Netherlands. ${ }^{5}$ Canisius Wilhelmina Hospital, PO Box 9015, 6500, GS, Nijmegen, The Netherlands. ${ }^{6}$ University Medical Centre Leiden, Albinusdreef 2, 2333, ZA, Leiden, The Netherlands. 'Erasmus Medical Centre, 's-Gravendijkwal 230, 3015, CE, Rotterdam, The Netherlands. ${ }^{8}$ Oslo University Hospital, P. O. Box 4950, Nydalen, N-0424 Oslo, Norway. ${ }^{9}$ Avicenna research institute Teheran, PO Box: 19615-1177, Teheran Postal code: 1936773493, Iran. ${ }^{10}$ Guy's hospital, Great maze pond, London SE1 9RT, UK. ${ }^{11}$ Birmingham women's hospital, Mindelsohn Way, Birmingham, West Midlands B15 2TG, UK. ${ }^{12}$ East Kent Hospitals University, Ethelbert road Canterbury, Kent CT1 3NG, UK. ${ }^{13}$ Catharina hospital, Michelangelolaan 2, 5623 EJ Eindhoven, the Netherlands. ${ }^{14}$ University of Illinois Hospital, 1740 W Taylor St, Chicago, IL 60612, USA. ${ }^{15}$ The Robinson Institute, School of Paediatrics and Reproductive Health, University of Adelaide, Adelaide, Australia.

Received: 5 February 2016 Accepted: 23 August 2018

Published online: 05 October 2018

\section{References}

1. Chan YY, Jayaprakasan K, Tan A, Thornton JG, Coomarasamy A, Raine-Fenning NJ. Reproductive outcomes in women with congenital uterine anomalies: a systematic review. [review]. Ultrasound Obstet Gynecol. 2011;38(4):371-82.

2. March CM. Hysteroscopy as an aid to diagnosis in female infertility. Clinical Obstetrics \& Gynecology. 1983;26(2):302-12.

3. Fedele L, Bianchi S, Marchini M, Franchi D, Tozzi L, Dorta M. Ultrastructural aspects of endometrium in infertile women with septate uterus. Fertility \& Sterility. 1996;65(4):750-2

4. Dabirashrafi H, Bahadori M, Mohammad K, Alavi M, Moghadami-Tabrizi N, Zandinejad K, Ghafari V. Septate uterus: new idea on the histologic features of the septum in this abnormal uterus. American Journal of Obstetrics \& Gynecology. 1995;172(1 Pt 1):105-7.

5. Raga F, Casan EM, Bonilla-Musoles F. Expression of vascular endothelial growth factor receptors in the endometrium of septate uterus. Fertility \& Sterility. 2009;92(3):1085-90.

6. Jaslow CR. Uterine factors. [review]. Obstet Gynecol Clin N Am. 2014;41(1):57-86.

7. Chan YY, Jayaprakasan K, Zamora J, Thornton JG, Raine-Fenning N, Coomarasamy $\mathrm{A}$. The prevalence of congenital uterine anomalies in unselected and high-risk populations: a systematic review. [review]. Hum Reprod Update. 2011;17(6):761-71.

8. Saravelos SH, Cocksedge KA, Li TC. Prevalence and diagnosis of congenital uterine anomalies in women with reproductive failure: a critical appraisal. [review] [95 refs]. Hum Reprod Update. 2008;14(5):415-29.

9. Homer HA, Li TC, Cooke ID. The septate uterus: a review of management and reproductive outcome. [review] [100 refs]. Fertility \& Sterility. 2000;73(1):1-14.

10. Nouri K, Ott J, Huber JC, Fischer EM, Stogbauer L, Tempfer CB. Reproductive outcome after hysteroscopic septoplasty in patients with septate uterus--a retrospective cohort study and systematic review of the literature. [review] [34 refs]. Reproductive Biology \& Endocrinology. 2010;8:52.

11. Valle RF, Ekpo GE. Hysteroscopic metroplasty for the septate uterus: review and meta-analysis. J Minim Invasive Gynecol. 2013;20(1):22-42.

12. Pang LH, Li MJ, Li M, Xu H, Wei ZL. Not every subseptate uterus requires surgical correction to reduce poor reproductive outcome. Int J Gynaecol Obstet. 2011;115(3):260-3.

13. Tonguc EA, Var T, Batioglu S. Hysteroscopic metroplasty in patients with a uterine septum and otherwise unexplained infertility. Int J Gynaecol Obstet. 2011;113(2):128-30.

14. Tomazevic T, Ban-Frangez H, Virant-Klun I, Verdenik I, Pozlep B, VrtacnikBokal E. Septate, subseptate and arcuate uterus decrease pregnancy and live birth rates in IVF/ICSI. Reprod BioMed Online. 2010;21(5):700-5.

15. Mollo A, De FP, Colacurci N, Cobellis L, Perino A, Venezia R, Alviggi C, De $P G$. Hysteroscopic resection of the septum improves the pregnancy rate of women with unexplained infertility: a prospective controlled trial. Fertility \& Sterility. 2009;91(6):2628-31.

16. Sugiura-Ogasawara M, Lin BL, Aoki K, Maruyama T, Nakatsuka M, Ozawa N, Sugi T, Takeshita T, Nishida M. Does surgery improve live birth rates in patients with recurrent miscarriage caused by uterine anomalies? Journal of obstetrics and gynaecology : the journal of the Institute of Obstetrics and Gynaecology. 2015;35(2):155-8.

17. Pabuccu R, Gomel V. Reproductive outcome after hysteroscopic metroplasty in women with septate uterus and otherwise unexplained infertility. Fertility \& Sterility. 2004;81(6):1675-8.
18. Christiansen OB, Nybo Andersen AM, Bosch E, Daya S, Delves PJ, Hviid TV, Kutteh WH, Laird SM, Li TC, van der Ven K. Evidence-based investigations and treatments of recurrent pregnancy loss. Fertil Steril. 2005;83(4):821-39.

19. Brigham SA, Conlon C, Farquharson RG. A longitudinal study of pregnancy outcome following idiopathic recurrent miscarriage. Human reproduction (Oxford, England). 1999;14(11):2868-71.

20. Kowalik CR, Goddijn M, Emanuel MH, Bongers MY, Spinder T, de Kruif JH, Mol BW, Heineman MJ. Metroplasty versus expectant management for women with recurrent miscarriage and a septate uterus. [review]. Cochrane Database Syst Rev. 2011;(6):CD008576.

21. Grimbizis GF, Gordts S, Di Spiezio SA, Brucker S, De AC, Gergolet M, Li TC, Tanos V, Brolmann H, Gianaroli L, et al. The ESHRE/ESGE consensus on the classification of female genital tract congenital anomalies. Hum Reprod. 2013;28(8):2032-44.

22. Grimbizis GF, Campo R. On behalf of the scientific Committee of the Congenital Uterine Malformations common Eshre/Esge working group: Stephan Gordts SBMGVITCLCDA: clinical approach for the classification of congenital uterine malformations. Gynecol Surg. 2012;9(2):119-29.

23. Graupera B, Pascual MA, Hereter L, Browne JL, Ubeda B, Rodriguez I, Pedrero C. Accuracy of three-dimensional ultrasound compared with magnetic resonance imaging in diagnosis of Mullerian duct anomalies using ESHREESGE consensus on the classification of congenital anomalies of the female genital tract. Ultrasound Obstet Gynecol. 2015;46(5):616-22.

24. Siam S, Soliman BS. Combined laparoscopy and hysteroscopy for the detection of female genital system anomalies results of 3,811 infertile women. J Reprod Med. 2014;59(11-12):542-6.

25. Ludwin A, Ludwin I, Kudla M, Pitynski K, Banas T, Jach R, Knafel A. Diagnostic accuracy of three-dimensional sonohysterography compared with office hysteroscopy and its interrater/intrarater agreement in uterine cavity assessment after hysteroscopic metroplasty. Fertil Steril. 2014;101(5):1392-9.

26. Faivre E, Fernandez H, Deffieux X, Gervaise A, Frydman R, Levaillant JM. Accuracy of three-dimensional ultrasonography in differential diagnosis of septate and bicornuate uterus compared with office hysteroscopy and pelvic magnetic resonance imaging. J Minim Invasive Gynecol. 2012;19(1):101-6.

27. Coccia ME, Becattini C, Bracco GL, Bargelli G, Scarselli G. Intraoperative ultrasound guidance for operative hysteroscopy a prospective study. J Reprod Med. 2000:45(5):413-8.

28. Regan LBM, Rai R. The investigation and treatment of couples with recurrent firsttrimester and second-trimester miscarriage. RCOG green-top guideline. 2011;17:1-18.

29. Goddijn M, van den Boogaard E, Steepers EA, Erwich JJ, Macklon NS, Land JA, Ankum WM. The guideline 'Recurrent miscarriage' (first revision) of the Dutch Society for Obstetrics and Gynaecology. Ned Tijdschr Geneeskd. 2008;152(30):1665-70.

30. Gaucherand P, Awada A, Rudigoz RC, Dargent D. Obstetrical prognosis of the septate uterus: a plea for treatment of the septum. [review] [25 refs]. European Journal of Obstetrics, Gynecology \& Reproductive Biology. 1994;54(2):109-12.

31. Valli E, Vaquero E, Lazzarin N, Caserta D, Marconi D, Zupi E. Hysteroscopic metroplasty improves gestational outcome in women with recurrent spontaneous abortion. Journal of the American Association of Gynecologic Laparoscopists. 2004;11(2):240-4.

32. Kirk EP, Chuong CJ, Coulam CB, Williams TJ. Pregnancy after metroplasty for uterine anomalies. Fertility \& Sterility. 1993;59(6):1164-8.

33. Maneschi F, Parlato M, Incandela S, Maneschi M. Reproductive performance in women with complete septate uteri. J Reprod Med. 1991;36(10):741-4.

34. Heinonen PK. Reproductive performance of women with uterine anomalies after abdominal or hysteroscopic metroplasty or no surgical treatment. Journal of the American Association of Gynecologic Laparoscopists. 1997;4(3):311-7.

35. Sugiura-Ogasawara M, Lin BL, Aoki K, Maruyama T, Nakatsuka M, Ozawa N, Sugi T, Takeshita T, Nishida M. Does surgery improve live birth rates in patients with recurrent miscarriage caused by uterine anomalies? J Obstet Gynaecol. 2015;35(2):155-8.

36. Ludwin A, Ludwin I. Comparison of the ESHRE-ESGE and ASRM classifications of Mullerian duct anomalies in everyday practice. Hum Reprod. 2015:30(3):569-80.

37. Smit JG, Overdijkink S, Mol BW, Kasius JC, Torrance HL, Eijkemans MJ, Bongers M, Emanuel MH, Veugels M, Broekmans FJ. The impact of diagnostic criteria on the reproducibility of the hysteroscopic diagnosis of the septate uterus: a randomized controlled trial. Hum Reprod. 2015;30(6):1323-30. 